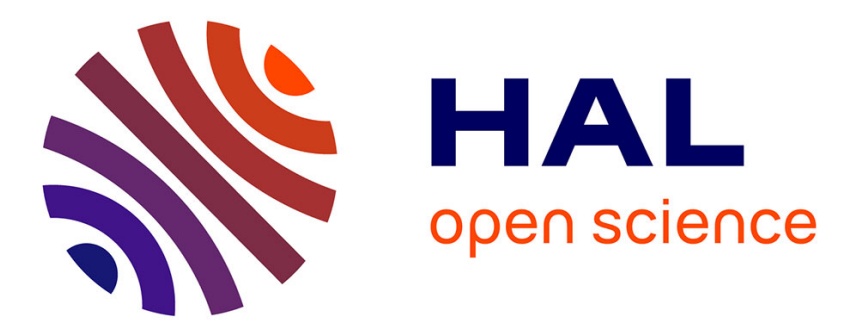

\title{
New constraints on the linear growth rate using cosmic voids in the SDSS DR12 datasets
}

\author{
Ixandra Achitouv
}

\section{To cite this version:}

Ixandra Achitouv. New constraints on the linear growth rate using cosmic voids in the SDSS DR12 datasets. Phys.Rev.D, 2019, 100 (12), pp.123513. 10.1103/PhysRevD.100.123513 . hal-02080710

\section{HAL Id: hal-02080710 https://hal.science/hal-02080710}

Submitted on 18 Jan 2022

HAL is a multi-disciplinary open access archive for the deposit and dissemination of scientific research documents, whether they are published or not. The documents may come from teaching and research institutions in France or abroad, or from public or private research centers.
L'archive ouverte pluridisciplinaire HAL, est destinée au dépôt et à la diffusion de documents scientifiques de niveau recherche, publiés ou non, émanant des établissements d'enseignement et de recherche français ou étrangers, des laboratoires publics ou privés. 


\title{
New constraints on the linear growth rate using cosmic voids in the SDSS DR12 datasets
}

\author{
I. Achitouv \\ Laboratoire Univers et Théories (LUTh), UMR 8102 Centre National de la Recherche Scientifique (CNRS), \\ Observatoire de Paris, Université Paris Diderot, 5 Place Jules Janssen, 92190 Meudon, France, \\ and Laboratoire Astroparticule et Cosmologie (APC), CNRS/IN2P3, Université Paris Diderot, \\ 75205 Paris Cedex 13, France
}

(Received 18 March 2019; published 10 December 2019)

\begin{abstract}
We present a new analysis of the inferred growth rate of cosmic structure measured around voids, using the LOWZ and the CMASS samples in the 12th data release (DR12) of SDSS. Using a simple multipole analysis we recover a value consistent with $\Lambda$ CDM for the inferred linear growth rate normalized by the linear bias: the $\beta$ parameter. We find $\beta=0.33 \pm 0.11$ for the LOWZ sample and $\beta=0.36 \pm 0.05$ for the CMASS sample. This work demonstrates that we can expect redshift-space distortions around voids to provide unbiased and accurate constraints on the growth rate, complementary to galaxy clustering, using simple linear modeling.
\end{abstract}

DOI: 10.1103/PhysRevD.100.123513

\section{INTRODUCTION}

The growth rate of cosmic structure $f$ tells us how fast density fluctuations $\Delta$ grow with respect to the scale factor of the Universe $a$ :

$$
f \equiv \frac{d \ln \Delta}{d \ln a},
$$

where $\Delta(r) \equiv 3 / r^{3} \int_{0}^{r} \delta_{\mathrm{DM}}\left(r^{\prime}\right) r^{\prime 2} d r^{\prime}$ and $\delta_{\mathrm{DM}}$ is the underlying dark matter density contrast. Its measurement as a function of time and scale is a key cosmological probe, very sensitive to the nature of gravity (e.g., $[1,2]$ ). To infer the growth rate, we can measure redshiftspace distortions (RSD) in the galaxy clustering signal. These distortions are due to the peculiar motions of galaxies, which on large scales have a coherent motion sourced by the gravitational potential of cosmic structures. This gravitational potential is itself proportional to the growth rate, in the linear regime. For standard general relativity and isotropic cosmologies, the linear growth rate does not depend on the comoving spatial scale [3] and can be approximated by $f \sim \Omega_{m}(z)^{\gamma}$, where $\Omega_{m}$ is the matter density parameter at redshift $z$, and $\gamma$ is a constant. For a $\Lambda$ CDM Universe $\gamma \sim 0.55$ [1,2], independently of the environment. Constraints on the linear growth rate made with galaxy-galaxy correlation function measurements in redshift space are well known, e.g., [4-9]. These measurements have shown a general consistency with the $\Lambda \mathrm{CDM}$ cosmological model, up to a $2.5 \%$ precision, albeit in some cases showing tension with the predictions of the latest cosmic microwave background measurements [10].
On the other hand, it was only recently that the growth rate has been inferred using the RSD pattern around cosmic voids. There are at least two reasons to perform this consistency test of the linear growth rate. First, certain models of modified gravity, such as $f(R)$ [11], rely on the chameleon screening mechanism [12] which suppresses the fifth force in high density regions, while in underdense regions the total gravitational force is enhanced (due to the presence of the fifth force), resulting in specific imprints on void abundance and density profiles around underdense regions (e.g., [13-18]). These theories would naturally lead to an environmentally dependent growth rate. In fact, in the nonlinear regime, the linear growth rate is also sensitive to the underlying density, as shown in [19]. For very large underdense regions, the effective cosmological parameters are expected to be different to the globally averaged parameters, but the quantification of this critical scale can also serve as an interesting test for departures from Einstein gravity. Second, the formation and evolution of cosmic voids is nonlinear but reduced compared to the dynamics of dark matter halos where overdense regions reach $\Delta(r) \gg 1$. This is why we can expect that quasilinear or linear models can describe the RSD around voids relatively well, although recent works have shown the limitation of this assumption [19-22]. The first studies that have tested the growth rate measurements using RSD around cosmic voids in galaxy surveys, have used a Gaussian streaming model (GSM) [3,21,23-25] to model the 2D galaxy-void correlation function in redshift space.

The analyses that first constrain the growth rate around voids from galaxy surveys are [26], where the authors used the CMASS sample of the Sloan Digital Sky Survey 
(SDSS), [27], where we used the low redshift 6-degree Field Galaxy Survey (6dFGS) [28] and [29], where the authors used the high redshift VIPERS survey datasets. While these analyses have shown an overall consistency with the $\Lambda \mathrm{CDM}$ expectation of the linear growth rate, the GSM does assume a knowledge of the real space density profiles around voids, which may induce a bias in the analysis. ${ }^{1}$ In [30] the authors took advantage of the approximated linear behavior of cosmic void evolution to perform a multipole analysis of the RSD around voids using both the CMASS and the LOWZ galaxy samples of SDSS DR12. Such a multipole analysis allows to derive the growth rate purely from the data measurement, assuming a linear relationship between the monopole and the quadrupole (see also the recent work of [31] for a complementary approach). With this assumption they have derived a linear growth rate consistent with $\Lambda \mathrm{CDM}$ in the CMASS sample, but at a $\sim 2-3 \sigma$ deviation from it in the LOWZ sample.

In this work we perform an independent analysis from [30] using a different void finder and a different treatment of the errors which enter into the likelihood analysis. Using the 500 mocks from the publicly available mock galaxy catalogs produced with the quick particle mesh (QPM) method [32], we test the validity of the multipole decomposition and use them to compute the covariance matrix that enters into the likelihood. We will show that in our case, we observe no deviation from $\Lambda \mathrm{CDM}$ when we disregard the multipole measurements at small scales $\left(<10 h^{-1} \mathrm{Mpc}\right)$.

We should also point out that in this work, similar to [27,29,30,33], we will neglect the Alcock-Paczynski effect [34]. This effect can be seen as geometrical distortions in the $2 \mathrm{D}$ void profiles that arise when transforming galaxy coordinates into comoving distances, using an underlying cosmological model that is incorrect. As it was also discussed in [35] the dominant contribution to the voidgalaxy quadrupole in redshift space comes from RSD rather than the Alcock-Paczynski effect. In what follows, we will indeed assume a $\Lambda \mathrm{CDM}$ cosmology to measure the galaxyvoid cross-correlation function. Hence we can see our final constraints on $\beta$ as a consistency test for the $\Lambda \mathrm{CDM}$ cosmology. Including the Alcock-Paczynski effect is beyond the scope of this work but we hope to take this effect into account in a future analysis.

This paper is organized as follows: in Sec. II we describe the data and the mocks we use to perform our analysis, in Sec. III we explain how we obtain our void catalogs, in Sec. IV we introduce the model we use to derive the linear growth rate, in Secs. V and VI we test our approach using the QPM mock catalogs and in the CMASS and LOWZ dataset. In Sec. VII we present our conclusion.

\footnotetext{
${ }^{1}$ However, one can marginalize over the void profiles fitted parameters [26].
}

\section{DATA AND MOCK CATALOGS}

We use the publicly available data of SDSS-III [36] data release 12 (DR12) which contains two datasets of galaxy catalogs from the Baryon Oscillation Spectroscopic Survey (BOSS): ${ }^{2}$ the LOWZ and the CMASS samples. Both map the southern and the northern hemispheres. The LOWZ north/south sample contains $\sim 248 / 114 \times 10^{3}$ galaxies in redshift range $0.15<z<0.43$ and a median $\bar{z}=0.32$ while the CMASS north/south sample contains $\sim 569 / 208 \times 10^{3}$ galaxies in redshift range $0.43<z<0.70$ with $\bar{z}=0.54$.

To identify the voids in the galaxy samples and to compute the multipoles, we use the two random catalogs generated by the BOSS collaboration (for each sample e.g., LOWZ north/south and CMASS north/south), featuring the redshift distribution. Each of these catalogs is referred to as RAN and RAN2. These random catalogs are also publicly available and contain about 50 times more points than the observed galaxies.

To compute the covariance matrix and to test our analysis, we use the publicly available SDSS-III DR12 mocks, generated with the quick particle mesh (QPM) algorithm [32]. They were generated assuming a flat $\Lambda \mathrm{CDM}$ cosmol$\operatorname{ogy}\left(\Omega_{\Lambda}=0.71, \Omega_{m}=0.29, \Omega_{b}=0.0458, \sigma_{8}=0.80, h=\right.$ $0.7, n_{s}=0.97$ ) [37]. We note that in these mocks, the linear galaxy bias is $b=2.2$ while in both CMASS and LOWZ it was estimated to be $b=1.85$ [37].

\section{VOID CATALOG}

To identify the cosmic voids in both the galaxy dataset and the QPM mocks, we use the void finder developed by [13], which was used in the 6dF Galaxy Survey analysis [27] to infer the growth rate. This void finder allows us flexibility in identifying the voids based on their density profiles $\delta(r)$ [13]. It uses a sample of RAN2 to identify candidate voids with an effective radius $r_{v}$ that satisfies the following density constraints: $\delta\left(r_{0}\right)<-0.9 ; \delta\left(r_{0}+d r\right)<$ $-0.8 ; \quad \delta\left(r_{0}+2 d r\right)<-0.3 ; \quad \delta\left(r_{v}\right)>0.15 ; \quad \delta\left(r_{v}+d r\right)>$ $\delta\left(r_{v}\right) ; \delta\left(r_{v}+d r\right)<0.4$, where the binning is given in steps of $d r=3 h^{-1} \mathrm{Mpc}, r_{0}=1.5 h^{-1} \mathrm{Mpc}$ and $\delta(r)$ is approximated by counting the number of galaxies around each random position we select from $\operatorname{RAN2}\left(D_{v}^{T} D_{g}\right)$, divided by the number of randoms we compute from the RAN catalogs $(R R)$ such that $\delta(r) \equiv D_{v}^{T} D_{g} / R R-1$. The ${ }^{T}$ index is to highlight that it is a test or candidate position for the voids and it is only marked as a void if the previous density conditions are satisfied. The first three conditions ensure that the center of the voids is underdense while the conditions around $r=r_{v}$ ensure that the selected voids have a ridge. We then perform two loops over these void candidates that satisfy the density conditions: the first loop to smooth the individual void profiles by requiring that

\footnotetext{
${ }^{2}$ http://www.sdss3.org/science/boss_publications.php.
} 


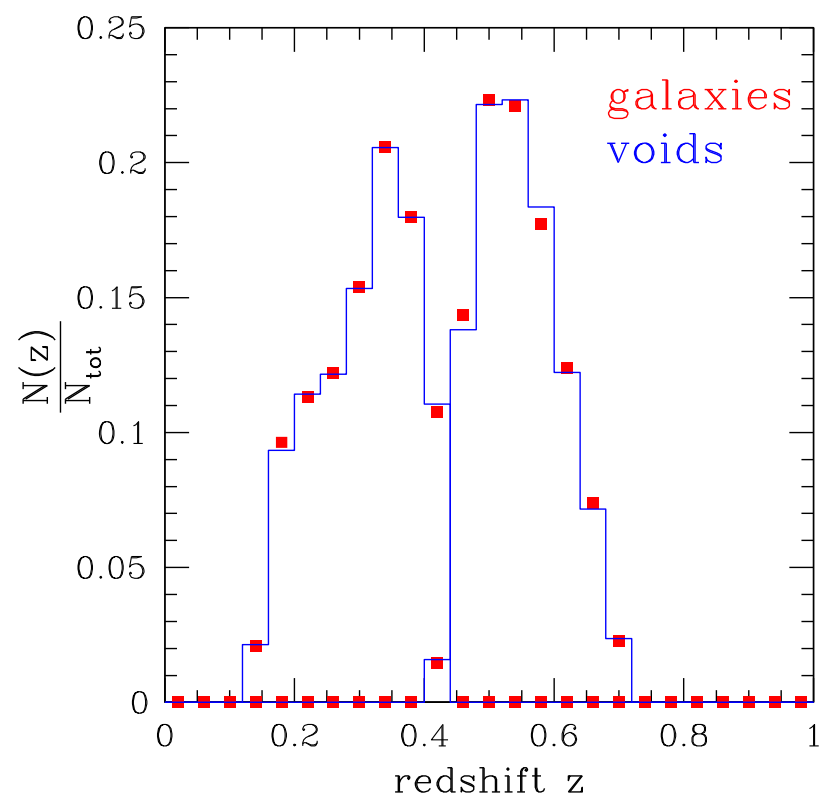

FIG. 1. Number of voids (blue histogram) and galaxies (red squares) in the LOWZ/CMASS samples, as a function of redshift normalized by the total number of voids/galaxies in each sample. Both overlap with one another as expected from the void finder.

$\delta\left(r_{0}+3 d r<r<r_{v} / 2\right)<-0.3$, the second to remove overlapping voids, keeping the largest.

We use 5 to 8 times more values than the galaxies/mock galaxies from the RAN2 catalog to identify the potential void candidates which is a good compromise between numerical computing power and having a convergence in the number of identified voids. Indeed, given that we remove overlapping voids, increasing the number of candidates can increase the number of identified voids up to a limited number. Keeping the same criteria for the data samples and the mocks, we end up with a selection of voids distributed in redshift as displayed in Fig. 1.

We repeat the same procedure using 500 QPM mocks for LOWZ North/South and CMASS North/South. Finally, we introduce a cut in the minimum size of the voids for the RSD analysis $r_{v}^{\min }=25 h^{-1}$ Mpc. The motivation for this cut is that (i) small voids identified with galaxy tracers do not necessarily correspond to underdensities in the matter density field. They also show a stronger deviation from linear evolution, and the galaxy bias around small voids can be amplified compared to the large-scale average bias [38-40]. (ii) We found that the overall void size distribution matches the mean value of the QPM mock distribution when $r>r_{v}^{\min }$. Although we are not interested in testing for void abundance in this work, having a mismatch in the void size distribution could introduce an offset between the mean void density profiles measured in the data and in the mocks, which could possibly introduce a bias in the derivation of our cosmological parameters. After applying this threshold, we found a total of 5986 voids identified in the LOWZ sample and 6373 in the CMASS sample.

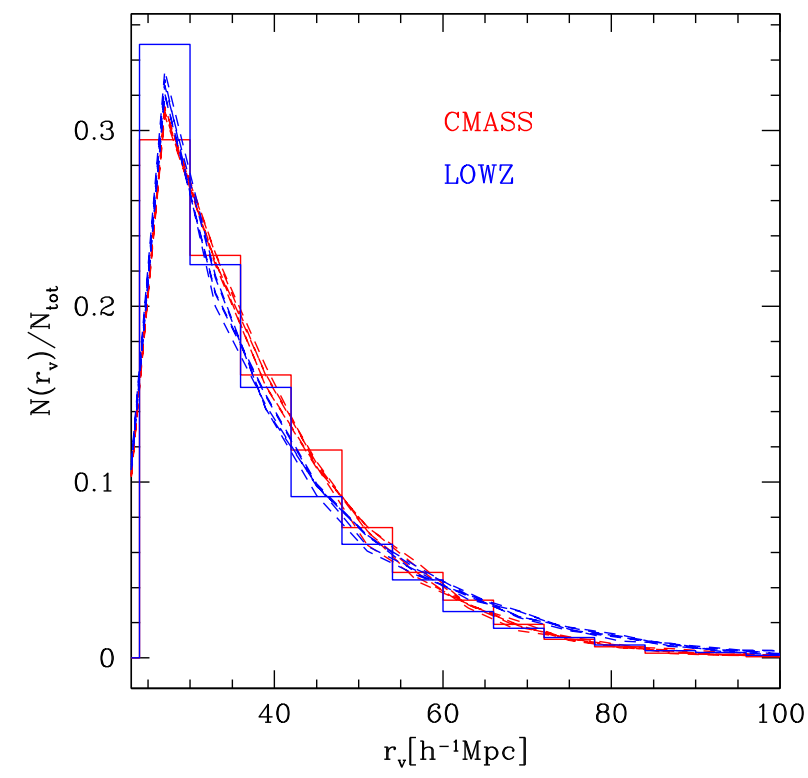

FIG. 2. Normalized number of voids as a function of the void radius in the LOWZ/CMASS samples (histograms) and in five randomly selected mocks (dashed curves). These probability density functions (PDFs) are qualitatively similar as a result of the void finder criteria, which remain unchanged in both samples.

The normalized number of voids as a function of radius is displayed in Fig. 2. The blue/red histograms correspond to the LOWZ/CMASS samples while the blue/red dashed curves correspond to five randomly selected samples from LOWZ/CMASS mocks, respectively. The mean void radius in the LOWZ/CMASS samples is, respectively, $r_{v}=38.5$ and $38 h^{-1}$ Mpc.

Finally we want to point out that, similar to our void finder, other void finders such as ZOBOV [41], have different criteria to select the voids depending on the "core-density threshold" (e.g., see discussion in Sec. III. 1 in [15]). A different choice of the parameters that identify the voids can result in different void catalogs which could have very different void density profiles. Therefore if one wants to change the void definition, a prior analysis on mock catalogs should ensure the pertinence of the void catalog by finding unbiased results of the cosmological parameters with respect to the fiducial cosmology of the mocks.

\section{METHODOLOGY}

\section{A. Multipole decomposition}

The peculiar velocities of galaxies, $\mathbf{v}$, which are due to the local gravitational potential result, on small scales, in random motions of galaxies within virialized halos. In principle this effect is not present within voids, which are generally empty of galaxies in their center. On large scales however, the coherent bulk flow pointing outwards from centers of voids is responsible for an overall coherent 
distortion known as the "Kaiser effect" [42]. It is this coherent outflow that carries information of the linear growth rate. Indeed, the galaxy peculiar velocities sourced by the underlying mass distribution of a void can be expressed in the linear regime as $([3,21,24,30])$

$$
\mathbf{v}(\mathbf{r})=\frac{-1}{3} \frac{f(z) H(z)}{1+z} \mathbf{r} \Delta(r),
$$

where $f(z)$ is the linear growth rate, $H(z)$ is the Hubble rate, $\mathbf{r} \equiv \mathbf{x}-\mathbf{X}$ is the separation between the comoving coordinate of the void center $\mathbf{X}$, and a galaxy at position $\mathbf{x}$. We also assume that on average the void density profiles are spherical and can be described by the density contrast $\Delta(r)$ where $r \equiv|\mathbf{r}|$. To relate the averaged galaxy density contract, $\bar{\xi}(r)$, to the matter density contrast, we generally assume a linear bias $b$ such that $\bar{\xi}(r)=b \Delta(r)$ and

$$
\bar{\xi}(r) \equiv \frac{3}{r^{3}} \int_{0}^{r} \xi(y) y^{2} d y,
$$

where $\xi(r)$ is equivalent to the galaxy density contrast at a scale $r$ (i.e., the galaxy-void cross-correlation function).

The peculiar velocity of a galaxy gives a contribution to the redshift space separation between the galaxy and the void center, and in the limit where $|\mathbf{r}| \ll \mathbf{X}$,

$$
\mathbf{s}=\mathbf{r}+\frac{(1+z) \hat{\mathbf{X}} \cdot v}{H(z)} \hat{\mathbf{X}}
$$

where $\hat{\mathbf{X}}$ is the unitary vector along the line of sight to our void center.

Performing a Jacobian transformation between the coordinate $\mathbf{s}$ and $\mathbf{r}$, at linear order, the redshift-space 2D correlation function can be described by $([21,30,42,43])$

$$
\xi^{s}(r, \mu)=\xi_{0}(r)+\frac{3 \mu^{2}-1}{2} \xi_{2}(r),
$$

where $\mu \equiv \cos (\theta)=\hat{\mathbf{X}}$. $\hat{\mathbf{r}}$ is the cosine of the angle between the line-of-sight direction and the separation vector while $\xi_{0}, \xi_{2}$ are the monopole and the quadrupole respectively, computed using the Legendre polynomials $P_{l}(\mu)$ via

$$
\xi_{l}(r)=\int_{0}^{1} \xi^{s}(r, \mu)(1+2 l) P_{l}(\mu) d \mu .
$$

In the linear regime [42],

$$
\begin{gathered}
\xi_{0}(r)=\left(1+\frac{\beta}{3}\right) \xi(r) \\
\xi_{2}(r)=\frac{2 \beta}{3}(\xi(r)-\bar{\xi}(r)),
\end{gathered}
$$

where $\beta=f / b$ and $\xi(r)$ is the real-space galaxy-void correlation function. These expressions lead to a simple relationship between monopole and quadrupole:

$$
\xi_{0}(r)-\bar{\xi}_{0}(r)-\xi_{2}(r) \frac{3+\beta}{2 \beta}=0 .
$$

This is the key equation that the authors of [30] have used to probe $\beta$ solely by measuring the monopole and the quadrupole. We will also use this equation in what follows, but we will introduce a cut at the scale $r_{\text {cut }}$ below which this approximation is no longer valid.

\section{B. Measurement of the galaxy-void correlation function}

To perform the multipole decomposition we start by measuring the void-tracer cross-correlation functions using the Landy-Szalay estimator:

$\xi_{v g}^{s}(r, \mu)=\frac{N_{r g} N_{r v}}{R_{v} R_{g}}\left(\frac{D_{v} D_{g}}{N_{g} N_{v}}-\frac{D_{g} R_{v}}{N_{g} N_{r v}}-\frac{D_{v} R_{g}}{N_{v} N_{r g}}\right)+1$,

where $D_{v} D_{g}$ is the number of data void-galaxy pairs, $R_{v} R_{g}$ the random void-galaxy pairs and $D_{g / v} R_{g / v}$ the number of galaxy/void data-random pairs, in bins at separation $r$ and $\mu$. The total number of galaxies, voids, galaxy randoms and void randoms are $N_{g}, N_{v}, N_{r g}$ and $N_{r v}$, respectively. In all cases we use a sample of the first random catalogs provided by the BOSS collaboration, having 10 times the number of galaxies/voids than our data samples.

We measure Eq. (8) in bins of $d \mu=0.045$ and $d r=4 h^{-1}$ Mpc. Then we perform the multipole decomposition using the Riemann integral.

\section{The likelihood analysis}

To infer the linear growth rate from the measurement of the monopole and quadrupole, we solve for the value of $\beta$ which satisfies Eq. (7), performing a Gaussian likelihood

$L\left(\xi_{0}, \xi_{2} \mid \beta\right)=\frac{1}{(2 \pi)^{N / 2} \sqrt{\operatorname{det} \mathbf{C}}} \exp \left[-\frac{1}{2} \sum_{i, j=l_{\min }}^{N} \varepsilon_{i} \mathbf{C}_{i j}^{-1} \varepsilon_{j}\right]$,

where the sum is in radial bins $r_{i}=\left[r_{\text {cut }}, r_{\text {max }}\right], \varepsilon_{i} \equiv$ $\xi_{0}\left(r_{i}\right)-\bar{\xi}_{0}\left(r_{i}\right)-\xi_{2}\left(r_{i}\right) \frac{3+\beta}{2 \beta}$ is the left-hand side of Eq. (7) and $\mathbf{C}$ is the covariance matrix $\mathbf{C}_{i j}=\left\langle\varepsilon_{i} \varepsilon_{j}\right\rangle$ which depends explicitly on $\beta$. Hence the normalization of the likelihood needs to be taken into account. Unlike the analysis performed in [30], which uses a jackknife method to estimate the covariance matrix, in what follows we compute the covariance matrix using 500 QPM mocks.

In Fig. 3 we show the correlation matrix (covariance matrix of the residuals after normalization by its diagonal) which can be compared to Fig. 4 in [30]. The correlations 

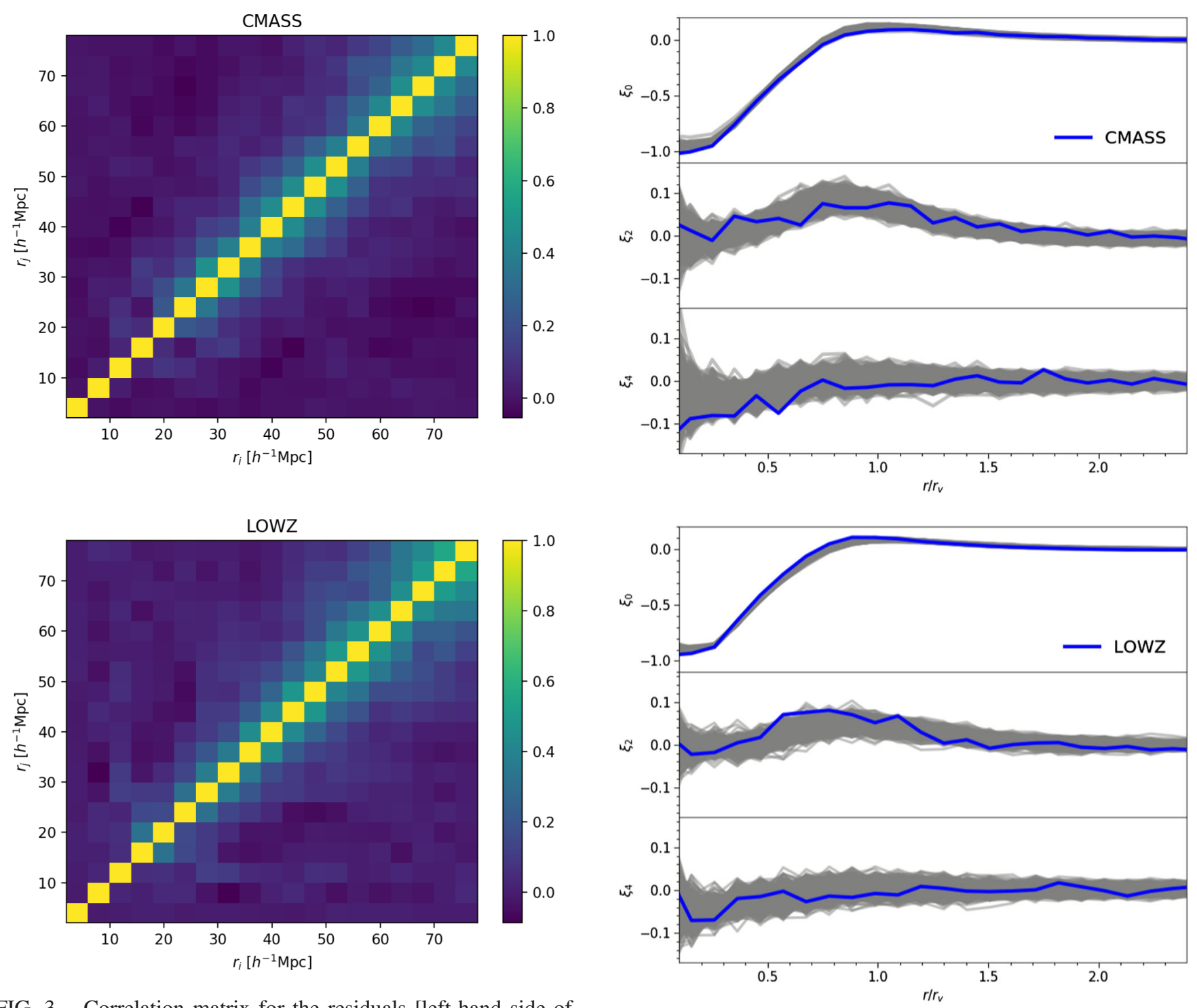

FIG. 3. Correlation matrix for the residuals [left-hand side of Eq. (7)], in the CMASS (top) and LOWZ (bottom) mocks using the best fit values of $\beta$ we obtained from the data (see Sec. VI).

between our bins follow the same qualitative trend as [30] for our voids of size $r_{v} \sim 38 h^{-1}$ Mpc: in the inner part of the voids, $r<r_{v}$, the bins seem less correlated while for $r>r_{v}$ we see some off diagonal correlations. We also note that in order to compute the galaxy-void correlation function we employ the Landy-Szalay estimator, while [30] uses the approximation $\xi_{l}(r) \simeq\left\langle D_{v} D_{g}\right\rangle-\left\langle D_{v} R_{g}\right\rangle$.

\section{ANALYSIS}

We start by using Eq. (8) to measure the galaxy-void correlation function in the data and the mocks, and then we apply Eq. (6) to compute the monopole $(l=0)$, quadrupole $(l=2)$ and hexadecapole $(l=4)$. The resulting multipoles are shown in Fig. 4, where the grey curves correspond to the mock measurements (1000 in total for CMASS and LOWZ) and the blue curves to the data. First we observe

FIG. 4. Multipole measurements in the mocks (grey curves) and in the data (blue curves) we obtained from Eq. (6). The data multipoles are qualitatively in good agreement with the ones we obtain in the mocks.

that the multipoles computed from the data and the mocks are qualitatively in good agreement with one another. Second we observe that for $r / r_{v} \leq 0.3$, which corresponds to a radius below $r \sim 10 h^{-1} \mathrm{Mpc}$, the slope of the monopole changes, and $\xi_{0} \rightarrow-1$ while $\left|\xi_{4}\right|>0$. These behaviors could indicate a breakdown of the linear assumptions and/ or ill-defined regions due to the lack of particle counts at the core of the voids. In any case, these low scales cannot be used within our current linear model hypothesis. Hence in what follows we define a cutoff scale $r_{\text {cut }}$ below which we disregard our measurements when performing the likelihood analysis.

Finally, we also show the measurement of the 2D galaxyvoid correlation function in both LOWZ/CMASS samples in Fig. 5, which we have measured parallel $(\pi)$ and 

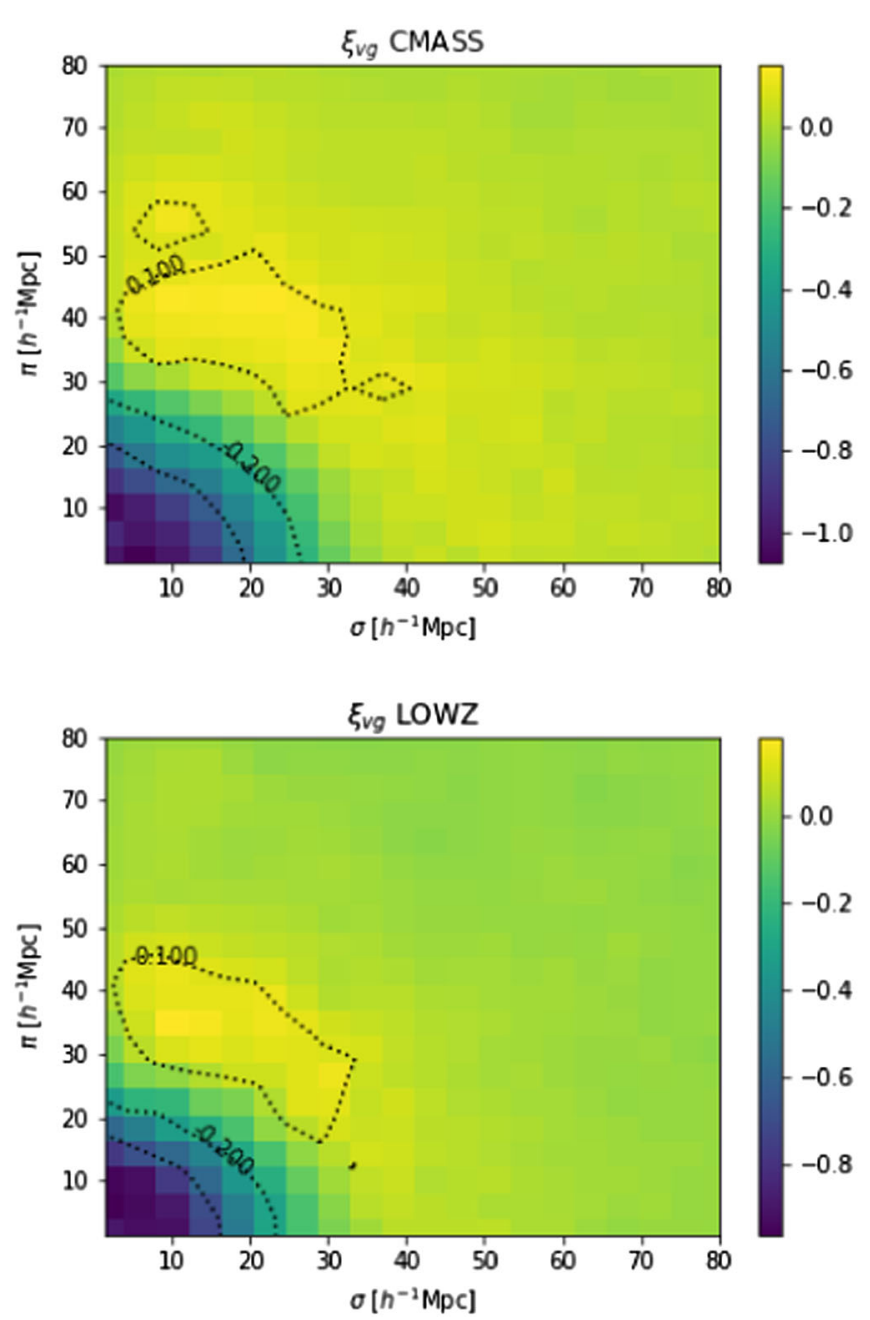

FIG. 5. The mean measurement of the 2D void-galaxy correlation function in CMASS (upper panel) and in LOWZ sample (lower panel). The dotted lines show isocontours of the data. Both measurements show apparent asymmetries that are characteristics of the coherent outflow of galaxies, sourced by the gravitational potential of the voids.

perpendicular $(\sigma)$ to the line of sight, using a binning of $4 h^{-1} \mathrm{Mpc}$. This is just to illustrate the asymmetry due to the peculiar velocities of galaxies that have a coherent outflow due to the gravitational potential of the void. This measurement could be used to extract the growth rate using a quasilinear modeling (e.g., Gaussian streaming model), as it was done in $[26,27,29]$. However it would require assumptions on the real space density profiles around the voids, which we know are sensitive to the underlying cosmology and to the void finder algorithm. Hence we do not explore further these 2D measurements.

\section{RESULTS}

In what follows, we set $r_{\text {cut }}=10 h^{-1} \mathrm{Mpc}$ and we use our measurement in bins of $d r=4 h^{-1} \mathrm{Mpc}$ up to $r_{\max }=78 h^{-1}$ Mpc. We have verified that the results we present in this section remain unchanged via the transformation $r_{\text {cut }} \rightarrow r_{\text {cut }} \pm d r$ or $r_{\max } \rightarrow r_{\text {max }} \pm d r$. We also tested that the inferred value of $\beta$ is not sensitive to the fiducial size of our voids $r_{v}$ nor to the hemisphere (splitting voids in large vs small, separating north vs south datasets). Thus for this analysis, we combined all the void sizes to obtain better statistical errors.

To obtain the best fit value for $\beta$, we use a large prior of $\beta=[-0.1,1.2]$ in steps of $d \beta=0.0024$. We have verified that our results remain unchanged by increasing the prior range.

\section{A. Mocks}

We start our analysis by inferring the value of $\beta$ on each individual mock catalog, using the likelihood computation given in Eq. (9) in order to evaluate the uncertainties on the $\beta$ measurement. In Fig. 6 we show the histogram of the best fit values we have found in the CMASS, LOWZ mocks as well as the mean values and the standard deviation: $\bar{\beta}=$ $0.36 \pm 0.06$ for the CMASS mocks and $\bar{\beta}=0.21 \pm 0.05$ for the LOWZ mocks. It is not trivial to compare these values to the mock expectations. Indeed, given the fiducial cosmology of the QPM mocks [32] we can easily compute the expected value for the growth rate but the linear bias is not explicitly given at the mean redshift of the mocks. At $z=0.5$ the linear bias is expected to be $b=2.2$ for the QPM mocks [36]. In such a case, we can extrapolate the value $\beta(z=0.5)=0.34$. This value can be compared to

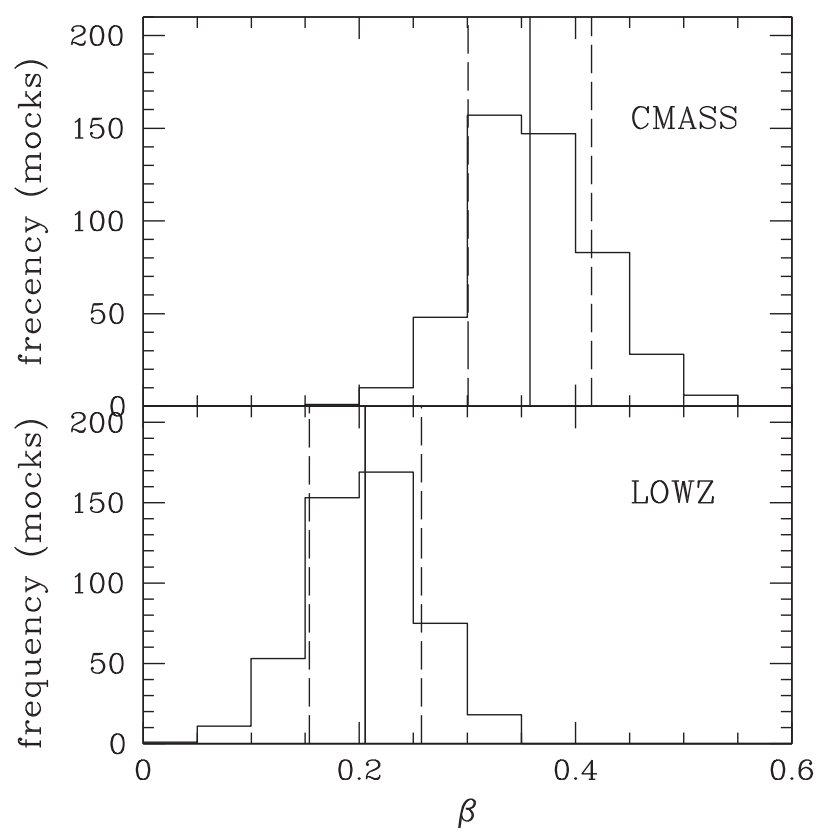

FIG. 6. Histogram of the inferred values of $\beta$ using the galaxyvoid multipole analysis in the QPM mocks. The solid lines correspond to the mean value $\bar{\beta}=0.36,0.21$ and the dashed lines to the $1-\sigma$ deviation $0.06,0.05$ for the CMASS, LOWZ samples, respectively. 
the CMASS mocks because in these mocks the redshift is $z \sim 0.54$. If we neglect the redshift dependence of the linear bias and keep $b=2.2$ but use the growth rate at the redshift of the LOWZ mocks then we can expect a value of $\beta=0.30$. Both theoretical values are within $2-\sigma$ deviation from the mean of $\beta$ we obtain. However, the errors on the mock mean give us $\bar{\beta}=0.36 \pm 0.003$ and $\bar{\beta}=0.21 \pm 0.002$. Hence we can argue that systematic errors are about $2 \%$ and $9 \%$ in the CMASS and LOWZ data. These differences are in agreement with the nonlinear evolution of the underlying matter density field: at low redshift, the linear model is more likely to have a higher systematic error compared to earlier times. On the other hand, we should make a few critical remarks:

(i) Galaxies around voids may be more biased compared to the average galaxies in the full simulation, in which case we can expect the fiducial value of $\beta$ to be lower than the one computed from $b=2.2$. We note however that in [27] the values of the linear bias we have inferred in mocks using the galaxy-void and the galaxy-galaxy correlation functions were consistent with one another. This must depend on the fiducial void size and the characteristics of the void profiles (e.g., amplitude at the void ridge).

(ii) We also point out the limitation of using QPM mocks [32] to test for the validity of the growth rate at low redshift. Indeed, unlike in full $\mathrm{N}$-body simulations, efficient algorithms such as [32] have not yet fully investigated the validity of their approach to reproduce the statistical description of the underdense matter density field.

Keeping these remarks in mind, we should adopt the more conservative approach: while $2 \%$ is below the statistical errors, $9 \%$ is on the other hand higher. Hence $a$ priori if we trust the QPM mocks then we should add quadratic systematic errors to the statistical errors when probing the data. We follow this approach in the next section with $\sigma_{\text {sys }}=2 \%, 9 \%$ for the CMASS, LOWZ data, respectively.

\section{B. Data}

Following the same procedure but for the data sample and adding up systematic errors in quadrature to the statistical errors, we find a best fit $\beta=0.33 \pm 0.11$ with $\sigma_{\text {stat }}=0.06$ and $\beta=0.36 \pm 0.05$ with $\sigma_{\text {stat }}=0.05$ for the LOWZ and CMASS samples, respectively, with a reduced $\chi^{2} /$ d.o.f. of $22.6 / 16=1.41$ and $21.8 / 16=1.36$. The probability Q that these $\chi^{2}$ values are due to chance are 0.1248 and 0.1497 , respectively. The posterior distribution is shown in Fig. 7. We note that our statistical errors on $\beta$ $\left(\sigma_{\text {stat }}\right)$ are consistent with what we found using the standard deviation of the best fit values from the mocks and that the best fit values correspond to the mean value of the likelihood PDF.

Once again we can compare these results with the expected values of $\beta$ in the case of a $\Lambda \mathrm{CDM}$ cosmology

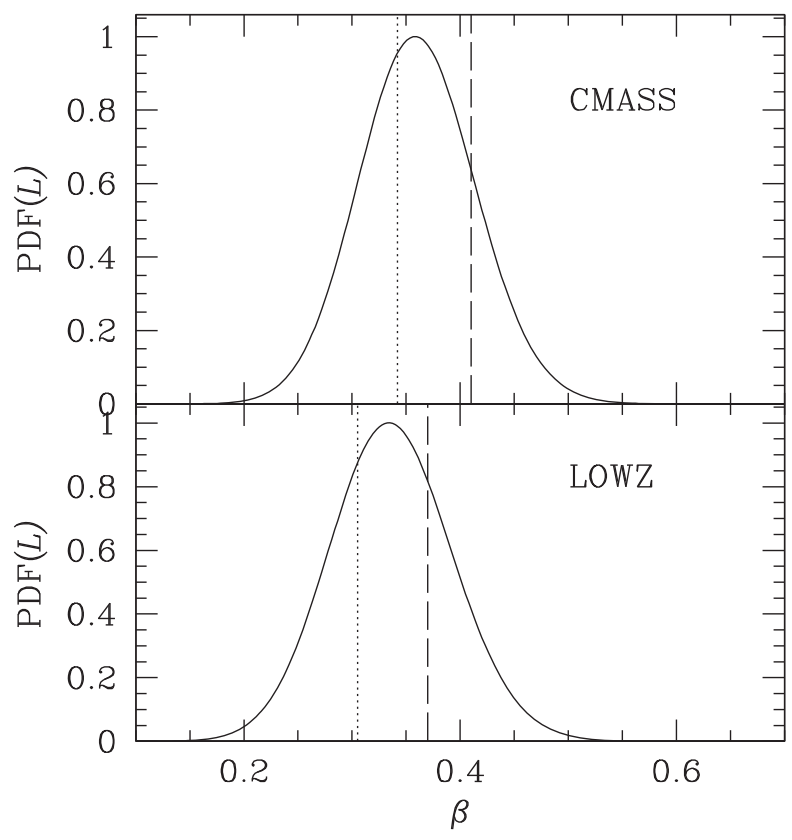

FIG. 7. Posterior distribution for $\beta$ in the CMASS and LOWZ data. The long-dashed line corresponds to the expected $\Lambda \mathrm{CDM}$ cosmology with a linear bias $b=1.85$ as it was inferred in [37] while the dotted line corresponds to the $\Lambda$ CDM cosmology with $b=2.2$ (i.e., the value of the linear bias in the QPM mocks at $z=0.5$ ).

(see Sec. II for cosmological parameter values). With a linear bias $b=1.85$ (as inferred in [37]), the theoretical values for LOWZ/CMASS are $\beta=0.37,0.41$ respectively. These are the same reference values that [30] have used to compare with their results. In Fig. 7 they correspond to the long dashed lines. Unlike what the authors in [30] have found, we obtain a 1- $\sigma$ agreement with respect to $\Lambda \mathrm{CDM}$, both for the LOWZ and the CMASS samples. We also show in Fig. 7 the fiducial values of $\beta$ for $b=2.2$ (motivated by the discussion in Sec. VIA). The latter is also consistent at $1-\sigma$ with our best fitting values (this is true even without including systematics errors).

\section{CONCLUSION}

In this work we have probed the parameter $\beta=f / b$, using the public galaxy catalogs released by the BOSS collaboration and an RSD multipole analysis of the galaxyvoid cross-correlation function. The model we used to infer the growth rate is derived from linear theory and was initially used in [30] to perform a similar analysis. However, in this work we find that our derived values for the growth rate are consistent with a $\Lambda \mathrm{CDM}$ cosmology within $1-\sigma$ in the data. Nevertheless, we introduce additional systematic errors that contribute significantly only for the LOWZ analysis. This errors is motivated by the analysis we realized upon the QPM mocks, showing a systematic error of $9 \%$ compared to the expected value of $\beta$ 
on the mock mean. Whether this systematic error is a result of the inappropriate linear multipole decomposition at low redshift or a failure of the QPM mocks to accurately match the nonlinear evolution of the underline matter density field is an open question which will require further investigation using full N-body simulations. In this work we adopt the conservative approach and enhance our error measurement by adding up the systematic and statistical errors in quadrature. Overall, the main differences in this analysis compared to the one presented in [30] are

(i) Our void catalogs are completely independent and based on different criteria (density criteria [13] vs watershed transform [44]). While the peak of the void size distribution is relatively similar in both studies, we have better statistics on the number of voids in the LOWZ sample. As a result, our statistical errors on $\beta$ are similar in both the LOWZ $\Delta \beta=0.06$ and CMASS sample $\Delta \beta=0.05$.

(ii) Motivated by our analysis with the mocks, we introduce a cut in scale to disregard our measurement at the center of the voids where $|\delta| \rightarrow-1$, which corresponds to the nonlinear regime where Eq. (7) does not hold in principle, as we discuss in Sec. V.

(iii) The treatment of the covariance matrix is different: in this work we used the mocks to compute the covariance while in [30] they used a jackknife method. We also provide in this work a complete study of the inferred values of $\beta$ within the mocks in order to check the validity of our model (Sec. VI A).

Overall, this work has provided some interesting results:

(i) Using mock catalogs, we have shown that $\beta$ can be extracted using no theoretical modeling of the voidgalaxy correlation function in real space. This is particularly interesting to avoid assuming a fiducial cosmology in order to predict the void density profiles, which could lead to potential bias of the growth rate value (the void density profiles carry the imprints of the cosmology e.g., [14,45-48]), or to avoid parametrizing the real space density profile and/or marginalizing over the profile parameters, which would introduce potentially weaker constraints on the growth rate.

(ii) The values of $\beta$ that we obtain in the LOWZ/ CMASS datasets are consistent with the value probed in [37]. However in [37] the scale range used to derive $\beta$ is $[40-180] h^{-1} \mathrm{Mpc}$, while we used the information contained within ranges $[10-78] h^{-1} \mathrm{Mpc}$. This illustrates again the complementarity of using cosmic voids to perform cosmological analysis: we have access to additional information, and the systematic errors are different. Finally we can emphasize the fact that the value of $\beta$ we obtained in this analysis is in good agreement with the $\Lambda \mathrm{CDM}$ linear prediction. It would be interesting to probe the information contained in smaller scales (e.g., below $10 h^{-1} \mathrm{Mpc}$ ) where the nonlinearities can carry more information. For instance, in [19] we have shown how the growth rate of cosmic structure can vary considerably when the underlying matter density $|\Delta| \geq 1$. We hope to perform such an analysis in future work.

\section{ACKNOWLEDGMENTS}

I am very grateful to Nico Hamaus and Cai Yanchuan for their useful discussions and for carefully proofreading this manuscript, giving me pertinent feedback. The research leading to these results has received funding from the European Research Council under the European Community Seventh Framework Programme (FP7/20072013 Grant Agreement No. 279954) RC-StG EDECS.
[1] D. Huterer et al., Growth of cosmic structure: Probing dark energy beyond expansion, Astropart. Phys. 63, 23 (2015).

[2] E. V. Linder, Cosmic growth history and expansion history, Phys. Rev. D 72, 043529 (2005).

[3] P. J. E. Peebles, Principles of Physical Cosmology (Princeton University Press, Princeton, 1993).

[4] F. Beutler, C. Blake, M. Colless, D. H. Jones, L. StaveleySmith, G. B. Poole, L. Campbell, Q. Parker, W. Saunders, and $\mathrm{F}$. Watson, The $6 \mathrm{dF}$ galaxy survey: $z \approx 0$ measurements of the growth rate and $\sigma_{8}$, Mon. Not. R. Astron. Soc. 423, 3430 (2012).

[5] C. Blake et al., The WiggleZ dark energy survey: Joint measurements of the expansion and growth history at $\mathrm{z}<1$, Mon. Not. R. Astron. Soc. 425, 405 (2012).
[6] S. de la Torre et al., The VIMOS public extragalactic redshift survey (VIPERS). Galaxy clustering and redshift-space distortions at $z \sim 0.8$ in the first data release, Astron. Astrophys. 557, A54 (2013).

[7] J. A. Peacock, A measurement of the cosmological mass density from clustering in the $2 \mathrm{dF}$ galaxy redshift survey, Nature (London) 410, 169 (2001).

[8] B. A. Reid et al., The clustering of galaxies in the SDSS-III baryon oscillation spectroscopic survey: Measurements of the growth of structure and expansion rate at $z=0.57$ from anisotropic clustering, Mon. Not. R. Astron. Soc. 426, 2719 (2012)

[9] M. Tegmark et al., Cosmological constraints from the SDSS luminous red galaxies, Phys. Rev. D 74, 123507 (2006). 
[10] P. A. R. Ade et al. (Planck Collaboration), Planck 2015 results. XIII. Cosmological parameters, Astron. Astrophys. 594, A13 (2016).

[11] W. Hu and I. Sawicki, Models of $f(R)$ cosmic acceleration that evade solar system tests, Phys. Rev. D 76, 064004 (2007).

[12] J. Khoury and A. Weltman, Chameleon cosmology, Phys. Rev. D 69, 044026 (2004).

[13] I. Achitouv, Testing the imprint of nonstandard cosmologies on void profiles using Monte Carlo random walks, Phys. Rev. D 94, 103524 (2016).

[14] I. Achitouv, M. Baldi, E. Puchwein, and J. Weller, Imprint of $\mathrm{f}(\mathrm{R})$ gravity on nonlinear structure formation, Phys. Rev. D 93, 103522 (2016).

[15] I. Achitouv, M. Neyrinck, and A. Paranjape, Testing spherical evolution for modelling void abundances, Mon. Not. R. Astron. Soc. 451, 3964 (2015).

[16] Y.-C. Cai, N. Padilla, and B. Li, Testing gravity using cosmic voids, Mon. Not. R. Astron. Soc. 451, 1036 (2015).

[17] J. Clampitt, Y.-C. Cai, and B. Li, Voids in modified gravity: Excursion set predictions, Mon. Not. R. Astron. Soc. 431, 749 (2013).

[18] P. Zivick, P. M. Sutter, B. D. Wandelt, B. Li, and T. Y. Lam, Using cosmic voids to distinguish $\mathrm{f}(\mathrm{R})$ gravity in future galaxy surveys, Mon. Not. R. Astron. Soc. 451, 4215 (2015).

[19] I. Achitouv and Y.-C. Cai, Modeling the environmental dependence of the growth rate of cosmic structure, Phys. Rev. D 98, 103502 (2018).

[20] I. Achitouv, Improved model of redshift-space distortions around voids: Application to quintessence dark energy, Phys. Rev. D 96, 083506 (2017).

[21] Y.-C. Cai, A. Taylor, J. A. Peacock, and N. Padilla, Redshiftspace distortions around voids, Mon. Not. R. Astron. Soc. 462, 2465 (2016).

[22] S. Nadathur and W. J. Percival, An accurate linear model for redshift space distortions in the void-galaxy correlation function, Mon. Not. R. Astron. Soc. 483, 3472 (2004).

[23] K. B. Fisher, On the validity of the streaming model for the redshift-space correlation function in the linear regime, Astrophys. J. 448, 494 (1995).

[24] N. Hamaus, P. M. Sutter, G. Lavaux, and B. D. Wandelt, Probing cosmology and gravity with redshift-space distortions around voids, J. Cosmol. Astropart. Phys. 11 (2015) 036.

[25] M. Kopp, C. Uhlemann, and I. Achitouv, Choose to smooth: Gaussian streaming with the truncated Zel'dovich approximation, Phys. Rev. D 94, 123522 (2016).

[26] N. Hamaus, A. Pisani, P. M. Sutter, G. Lavaux, S. Escoffier, B. D. Wandelt, and J. Weller, Constraints on Cosmology and Gravity from the Dynamics of Voids, Phys. Rev. Lett. 117, 091302 (2016).

[27] I. Achitouv, C. Blake, P. Carter, J. Koda, and F. Beutler, Consistency of the growth rate in different environments with the 6-degree field galaxy survey: Measurement of the void-galaxy and galaxy-galaxy correlation functions, Phys. Rev. D 95, 083502 (2017)

[28] D. H. Jones et al., The $6 \mathrm{dF}$ galaxy survey: Samples, observational techniques and the first data release, Mon. Not. R. Astron. Soc. 355, 747 (2004).
[29] A. J. Hawken et al., The VIMOS public extragalactic redshift survey: Measuring the growth rate of structure around cosmic voids, Astron. Astrophys. 607, A54 (2017).

[30] N. Hamaus, M.-C. Cousinou, A. Pisani, M. Aubert, S. Escoffier, and J. Weller, Multipole analysis of redshift-space distortions around cosmic voids, J. High Energy Phys. 07 (2017) 014

[31] C. M. Correa, D. J. Paz, N. D. Padilla, A. N. Ruiz, R. E. Angulo, and A. G. Sánchez, Non-fiducial cosmological test from geometrical and dynamical distortions around voids, Mon. Not. R. Astron. Soc. 485, 5761 (2019).

[32] M. White, J. L. Tinker, and C. K. McBride, Mock galaxy catalogues using the quick particle mesh method, Mon. Not. R. Astron. Soc. 437, 2594 (2014).

[33] A. J. Hawken, M. Aubert, A. Pisani, M.-C. Cousinou, S. Escoffier, S. Nadathur, G. Rossi, and D. P. Schneider, Constraints on the growth of structure around cosmic voids in eBOSS DR14, arXiv:1909.04394.

[34] C. Alcock and B. Paczynski, An evolution free test for nonzero cosmological constant, Nature (London) 281, 358 (1979).

[35] S. Nadathur, P. M. Carter, W. J. Percival, H. A. Winther, and J. E. Bautista, Beyond BAO: Improving cosmological constraints from BOSS data with measurement of the void-galaxy cross-correlation, Phys. Rev. D 100, 023504 (2019).

[36] S. Alam et al., The clustering of galaxies in the completed SDSS-III baryon oscillation spectroscopic survey: Cosmological analysis of the DR12 galaxy sample, Mon. Not. R. Astron. Soc. 470, 2617 (2017).

[37] C.-H. Chuang et al., The clustering of galaxies in the completed SDSS-III Baryon Oscillation Spectroscopic Survey: Single-probe measurements from DR12 galaxy clustering-towards an accurate model, Mon. Not. R. Astron. Soc. 471, 2370 (2017).

[38] G. Pollina, N. Hamaus, K. Dolag, J. Weller, M. Baldi, and L. Moscardini, On the linearity of tracer bias around voids, Mon. Not. R. Astron. Soc. 469, 787 (2017).

[39] G. Pollina et al., On the relative bias of void tracers in the dark energy survey, Mon. Not. R. Astron. Soc. 487, 2836 (2019).

[40] P. M. Sutter, G. Lavaux, N. Hamaus, B. D. Wandelt, D. H. Weinberg, and M.S. Warren, Sparse sampling, galaxy bias, and voids, Mon. Not. R. Astron. Soc. 442, 462 (2014).

[41] M. C. Neyrinck, ZOBOV: A parameter-free void-finding algorithm, Mon. Not. R. Astron. Soc. 386, 2101 (2008).

[42] N. Kaiser, Clustering in real space and in redshift space, Mon. Not. R. Astron. Soc. 227, 1 (1987).

[43] A. J. S. Hamilton, Introduction and Preface, in The Evolving Universe, edited by D. Hamilton, Astrophysics and Space Science Library, Vol. 231 (Springer, Dordrecht, 1998), pp. 1-10.

[44] P. M. Sutter, G. Lavaux, N. Hamaus, A. Pisani, B. D. Wandelt, M. Warren, F. Villaescusa-Navarro, P. Zivick, Q. Mao, and B. B. Thompson, VIDE: The void identification and examination toolkit, Astron. Comput. 9, 1 (2015).

[45] E. Adermann, P. J. Elahi, G. F. Lewis, and C. Power, Cosmic voids in evolving dark sector cosmologies: The high-redshift universe, Mon. Not. R. Astron. Soc. 479, 4861 (2018). 
[46] M. Cautun, E. Paillas, Y.-C. Cai, S. Bose, J. Armijo, B. Li, and N. Padilla, The Santiago-Harvard-EdinburghDurham void comparison-I. Shedding light on chameleon gravity tests, Mon. Not. R. Astron. Soc. 476, 3195 (2018).

[47] C. D. Kreisch, A. Pisani, C. Carbone, J. Liu, A. J. Hawken, E. Massara, D. N. Spergel, and B. D. Wandelt, Massive neutrinos leave fingerprints on cosmic voids, Mon. Not. R. Astron. Soc. 488, 4413 (2019).

[48] E. Paillas, M. Cautun, B. Li, Y.-C. Cai, N. Padilla, J. Armijo, and S. Bose, The Santiago-Harvard-Edinburgh-Durham void comparison II: Unveiling the Vainshtein screening using weak lensing, Mon. Not. R. AstrHVon. Soc. 484, 1149 (2019). 